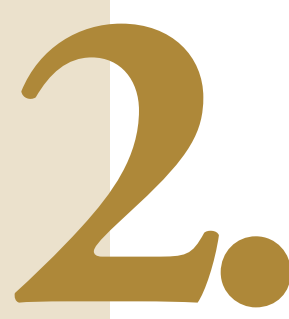

La Calidad Académica, un Compromiso Institucional

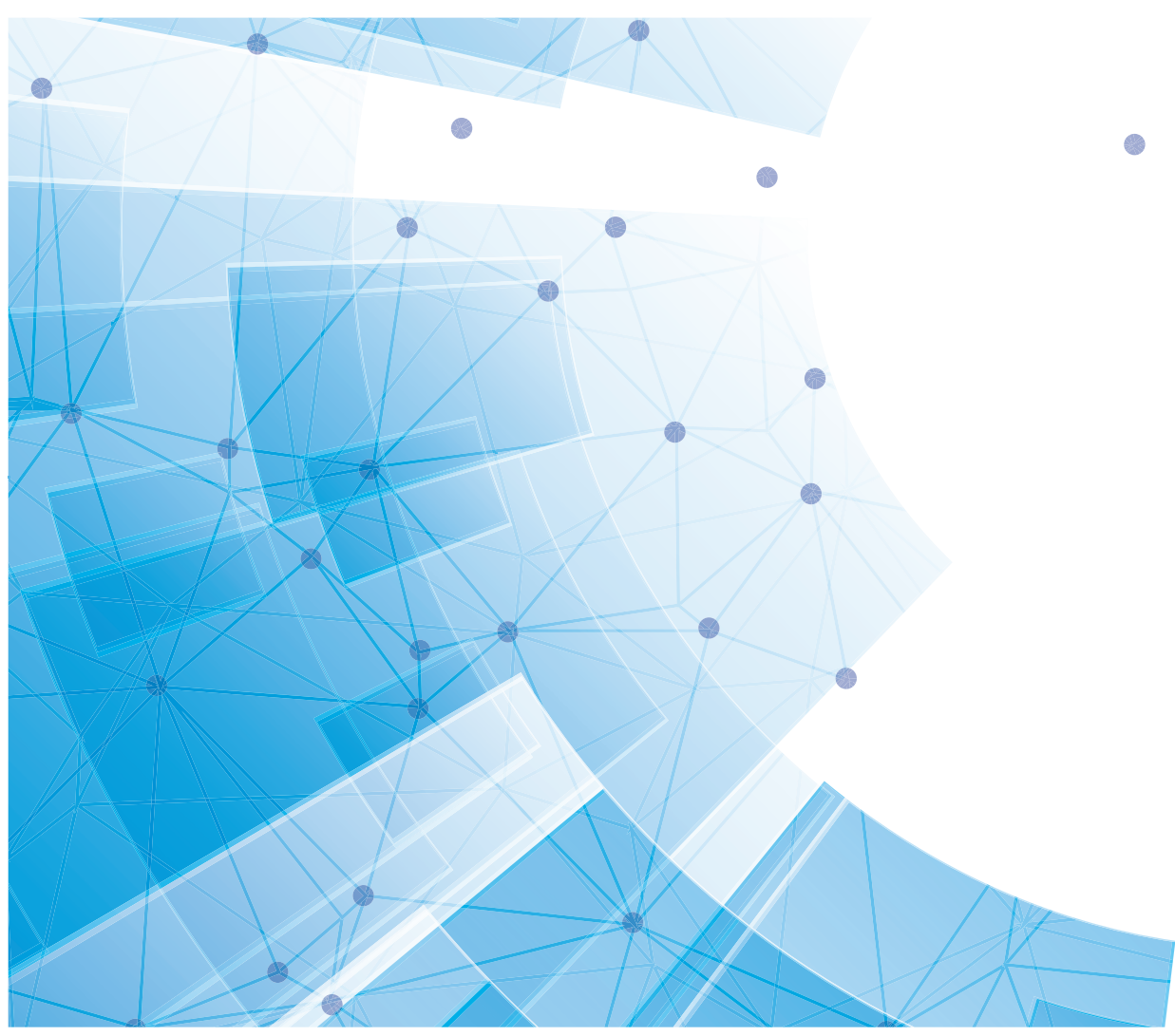

Propuesta de un modelo deterministico para la

Avellaneda Bautista Campo Alcides,

Borda Arias María Isabel (2018).

Propuesta de un modelo

determnístico para la

estimación de la fideli-

dad de la información

contable.

Criterio Libre, 16 (28),

61-84

ISSN 1900-0642

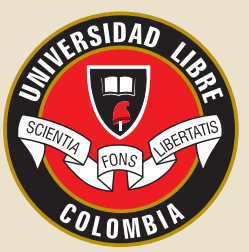
(n)

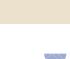




\title{
PROPUESTA DE UN MODELO DETERMINÍSTICO PARA LA ESTIMACIÓN DE LA FIDELIDAD DE LA INFORMACIÓN CONTABLE*
}

\author{
PROPOSAL OF A DETERMINISTICAL MODEL FOR THE \\ ESTIMATION OF THE FIDELITY OF THE \\ ACCOUNTING INFORMATION \\ PROPOSTA DE UM MODELO DETERMINÍSTICO PARA A \\ ESTIMATIVA DA FIDELIDADE DA INFORMAÇÃO CONTÁBIL \\ UNE PROPOSITION D'UN MODÈLE DETERMINISTE POUR \\ L'ESTIMATION DE LA FIDÉLITÉ DE L'INFORMATION COMPTABLE
}

\section{CAMPO ALCIDES AVELLANEDA BAUTISTA, MSC" MARÍA ISABEL BORDA ARIAS, PHD***}

Fecha de Recepción: 23 de enero de 2018 Fecha de aprobación: 25 de mayo de 2018

RESUMEN

La función misional de la contabilidad es generar información relacionada con fenómenos patrimoniales. Uno de sus más valiosos atributos, la fidelidad o característica que garantiza que la información objeto representa lo que pretende representar, ha sido puesta en tela de juicio debido a los escándalos financieros ocurridos durante el período 1991-2016. En ellos se evidencia manipulación de esa información y, por consiguiente, han minado la confianza de los usuarios. Sin embargo, no es visible un sistema objetivo para determinar el grado en que un cuerpo informacional cumple o incumple con dicho atributo. Se propone aquí un modelo para medir el acercamiento de un cuerpo informacional a la realidad que pretende representar.

* Artículo producto de investigación.

** Contador público, magíster en contabilidad; magíster en educación, especialista en revisoría fiscal. Especialista en ciencias tributarias. Académico certificado (ANFECA). Profesor investigador, Universidad Libre, Bogotá, Colombia; líder Grupo de investigación Constructores Contables.

*** Psicóloga; magíster en psicología organizacional y del trabajo; especialización en psicología clínica; PhD en administración. Profesora investigadora, Universidad Libre, Bogotá, Colombia. Investigadora en el Grupo de investigación Constructores Contables.

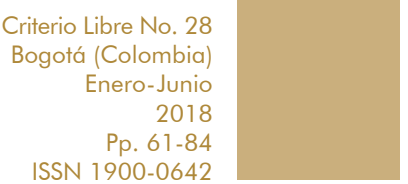




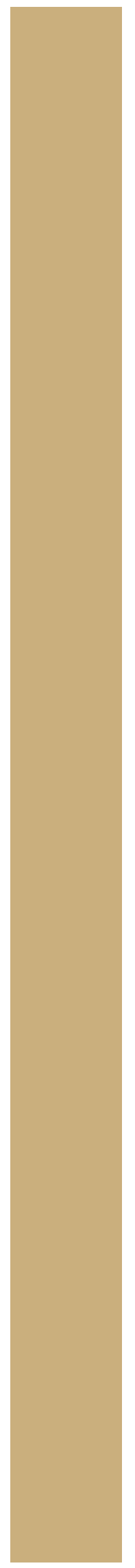

PALABRAS CLAVE:

Agentes sociales, fidelidad, información contable, medición.

CLASIFICACIÓN JEL:

C 44, M 14, M 41, y M 42.

ABSTRACT

The missionary function of accounting is to generate information related to heritage phenomena. One of its most valuable attributes, fidelity or the characteristic that guarantees that the object information represents what it intends to represent, has been questioned due to the financial scandals that occurred during the 1991-2016 period. In them, manipulation of that information is evidenced and, consequently, they have undermined the confidence of the users. However, an objective system is not visible to determine the degree to which an informational body meets or fails to comply with the attribute mentioned. A model is proposed here to measure the approach of an informational body to the reality that it tries to represent.

Key words: accounting information, fidelity, measurement, social agents.

JEL Classification: C 44, M 14, M 41, y M 42.

\section{RESUMO}

A função misional da contabilidade é gerar informação relacionada com fenômenos patrimoniais. Um dos mais valiosos atributos, a fidelidade ou característica que garante que a informação objeto representa o que busca representar, foi questionado devido aos escândalos financeiros aconteceu durante o período 1991-2016. Neles é comprovada manipulação daquela informação e, por conseguinte, eles minaram a confiança dos usuários. Porém, não é visível um sistema objetivo á determinar o grau dentro que um corpo informacional comple ou incumple com este atributo. Pretende-se aqui um modelo á medir a aproximação de um corpo informacional para a realidade que busca representar.

Palavras chave: agentes sociais, fidelidade, informação contável, medição.

Classificação JEL: C 44, M 14, M 41, y M 42. 
La fonction de mission de la comptabilité est de générer d'information relative aux phénomènes patrimoniaux. L'un de ses attributs les plus précieux, la fidélité ou la caractéristique qui garantit que l'information l'objet représente ce qu'elle essaie de représenter, a été mise à un tissu de jugement grâce aux scandales financiers arrivés durant la période 1991-2016. Dans ceuxci une manipulation de cette information est manifeste et, par conséquent, elles ont miné la confiance des utilisateurs. Cependant, un système objectif n'est pas visible pour déterminer le degré dans lequel un corps informacional s'acquitte ou faut avec le dit attribut. Un modèle se propose ici pour mesurer le rapprochement d'un corps informacional à la réalité qu'elle essaie de représenter.

Mots clés: agents sociaux, fidélité, information comptable, mesurage.

Classification JEL: C 44, M 14, M 41, y M 42.

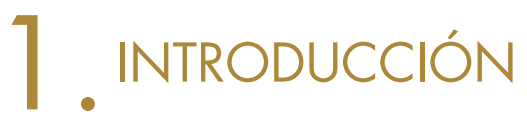

En este trabajo se propone un modelo determinístico de indicadores para estimar el grado de acercamiento de una información contable y financiera a su nivel de fidelidad, con la pretensión de contribuir a fortalecer los sistemas de aseguramiento y la confianza pública, misma que ha sido debilitada a raíz de los frecuentes escándalos financieros ocurridos durante los recientes veinticinco años en los que la contabilidad estuvo entre los principales protagonistas.

Referente al concepto de fidelidad de la información, el Marco Conceptual de las Normas Internacionales de Información Financiera (NIIF) dice que una de sus características más relevantes es la Representación fiel, pues ella "debe representar fielmente los fenómenos que pretende representar" (IIFRS Foundation, 2010, p. 13).

Ya que el público (conjunto de los agentes sociales) no tiene acceso directo a las instalaciones de la organización difusora para verificar la existencia real de lo que se quiere representar en la información, su confianza en el ente depende de esa fidelidad en la información.

Pareciera que una de las razones que han contribuido a debilitar la seguridad de la información contable es el hecho de que la objetividad que orientaba su razón de ser desde finales del siglo XIX fue remplazada por un normativismo emergido desde los mercados financieros e instalado de manera definitiva en la regencia de la disciplina contable, mediante un proceso ocurrido en la década de 1990, el cual permite una significativa flexibilización en los sistemas de control y aseguramiento. 
Actualmente la información contable y financiera que difunden las organizaciones es preparada conforme a normas y principios originados en las sanas prácticas, como es el caso de las Normas Internacionales de Información Financiera, NIIF, las Normas Internacionales de Auditoría, NIA (Federación Internacional de Contadores, $2012)$ y las Normas de Aseguramiento de la Información, NIAS, acogidas como políticas públicas por muchos gobiernos, incluido el de Colombia (Gobierno de Colombia, Presidencia de la República, 2009).

Sin embargo, coincidencialmente, en el mismo período antes referido los medios de comunicación hicieron despliegue más que suficiente acerca de los escándalos financieros ocurridos en el mundo durante el período 1991 a 2016 . En ellos ha estado como en el ojo del huracán la información contable manipulada, distorsionada, destruida u ocultada. Es decir, alejada de la fidelidad que debiera tener.

Ante tal problemática, los gobiernos de muchos países y organismos multilaterales relacionados con la economía propenden por establecer reglas que permitan prevenir riesgos y establecer mejores controles a la información. Tal es el caso de la promulgación de la Ley Sarbanes-Oxley con la cual Estados Unidos intenta recuperar la confianza pública y poner más controles a las organizaciones.

Colombia, con la Ley 1314 de 2009, para estar a tono con la internacionalización de la economía prescribió un nuevo orden para la contabilidad basado en la convergencia a estándares internacionales. En ella se explicita el desarrollo del aseguramiento de la información enfocado en las normas de control y de ética establecidos por IFAC (sigla en inglés).

No obstante esos esfuerzos, continúan los escándalos por la caída de grandes organizaciones que en su momento fueron dignas de toda credibilidad y se repiten los casos en los que la información difundida no representa lo que se supone que debe representar.
Teniendo en cuenta que la función natural de la contabilidad es emitir información confiable para la toma de decisiones, que el principal atributo de esa información es la fidelidad de los hechos que ella representa, que ese atributo ha sido reiteradamente vulnerado y que la evaluación basada en controles normativos no ha sido suficientemente eficaz porque el riesgo continúa, surge la pregunta:

¿Cómo sería un sistema eficaz que permita a los agentes sociales confiar en que una información contable representa fielmente los fenómenos que pretende representar?

El objetivo del presente trabajo es elaborar al menos un indicador para calcular con eficacia el grado de fidelidad que se cumple en un conjunto de información contable y financiera.

Para lograr el objetivo general se requiere: a) describir el principio de autonomía de la información contable, b) relacionar la fidelidad de la información con la objetividad generada por el principio de autonomía de la información contable, c) construir un indicador que permita medir el grado de fidelidad que pudiera tener un cuerpo de información contable con relación a los hechos que pretende representar, d) comprobar la eficacia del indicador de fidelidad mediante su aplicación a un conjunto de información contable.

El sistema propuesto es útil para:

- Tomar decisiones más seguras en materia de dinámicas patrimoniales por parte de los evaluadores de información contable lauditores, revisores fiscales, jueces de la república, entidades fiscalizadoras y los agentes sociales en general.

- Identificar oportunamente las asimetrías de la información, y se pueden diseñar evaluaciones prospectivas que permitan minimizar los riesgos patrimoniales por el mal uso de la información contable y financiera.

- Ampliar el espectro de la contabilidad como cuerpo de conocimientos, y simplificar el trabajo evaluativo en el aseguramiento de 
la información por parte de los profesionales contables.

- Fortalecer el valor cualitativo de la información contable y, por tanto, aumentar la importancia de la contabilidad como bien social.

El sistema propuesto puede llegar a ser utilizado como herramienta científica en la evaluación de la fidelidad de la información. Por otra parte, definir un sistema objetivo de medida para indicar el grado de fidelidad que se puede conceder a un conjunto de información contable, fortalece la confianza pública.

\section{MÉTODO Y PROBLEMA}

En esta investigación se siguió el enfoque mixto (cualitativo-cuantitativo), se aplicó la lógica formal y la lógica matemática. La recolección de la información se hizo por consulta de fuentes secundarias y se acumuló en tres tipos de archivos, así: fichas académicas, conformación de una de bases de datos y se conformó un archivo de recopilación documental. En el procesamiento de la información predominó el proceso analíicico deductivo con acercamiento al modelaje determinístico.

Se tomó como foco problémico la inseguridad en el manejo de la información contable y financiera, se trató como evidencia empírica los principales escándalos financieros ocurridos en el mundo, información que fue difundida por los diferentes medios de comunicación; hechos de los cuales se consideró como muestra representativa seis de esos casos ocurridos durante el período $1992-2016$.

Los referidos escándalos pusieron en tela de juicio la contabilidad y su principal producto, la información, debido a que en la mayoría de esos escándalos hubo manipulación, omisión y otras maniobras fraudulentas contra la fidelidad de la información contable y financiera, como se evidencia en los siguientes casos que constituyen apenas algunos de los más sonados.

Banco Morgan. Principal oficina en Londres. "Merril y Morgan Conocían del crítico estado del Fondo LTCM, y es que ambas entidades examinaron los libros contables del fondo de cobertura un mes antes de que se anuncia el paquete de rescate reunido por 14 poderosas instituciones financieras, en las que estaban incluidas Merril Lynch y JP Morgan" (Corrigan \& Lewis, 1998). En este caso el origen del fraude fue que hubo ocultamiento de información.

Enron (2001). Con sede en Houston, Texas. El País desplegó una amplia información sobre el caso Enron. Se citan tres apartes de la noticia:

"el mayor escándalo financiero vivido por el país norteamericano hasta el momento ha llenado páginas y páginas en los periódicos de todo el mundo desde que comenzó a salir a la luz".

"Comienza la investigación sobre el caso por Arthur Andersen, la auditora de la energética".

"El jurado encuentra culpable a Andersen por obstrucción a la justicia en 'caso Enron' por destruir documentos sobre pérdidas de más de mil millones de dólares en la compañía que auditaba. El veredicto supondrá el fin de la auditora, fundada 89 años antes en Chicago" (El País.es, 2006).

Laboratorio Merck, Norteamérica. A mediados del año 2002 los medios publicaban el escándalo ocurrido en la prestigiosa firma, de la siguiente manera: "La farmacéutica Merck se convirtió hoy en la última empresa estadounidense en sumarse a la lista de compañías que se han visto envueltas en escándalos contables, al anunciar que contabilizó como ventas unos 14.060 millones de dólares que nunca existieron" (EFE, El Tiempo, 2002).

Parmalat. Compañía italiana; en el año 2004 los medios, entre ellos El País, publicaban el escándalo de la reconocida organización así: "Crisis de confianza. Parmalat llevaba en secreto una contabilidad cuádruple. El balance que contiene la verdadera situación patrimonial de 
Parmalat fue recuperado del ordenador del ex auditor interno del grupo, (...)" (El País, 2004).

Bogotá, Colombia. Carrusel de la Contratación (2010). Una de las formas en que los medios difundieron el escándalo fue así:

"El escándalo sobre el llamado 'Carrusel de la Contratación' estalló el 25 de junio del año pasado, cuando Caracol Radio reveló en exclusiva una grabación que involucraba al Contralor de Bogotá con la presunta negociación de omisiones con contratistas" (Caracol.com/Radio, 2011 ).

Los referidos escándalos pusieron en tela de juicio la contabilidad y su principal producto, la información, debido a que en la mayoría de esos escándalos hubo manipulación, omisión y otras maniobras fraudulentas contra la fidelidad de la información contable y financiera, como se evidencia en los siguientes casos que constituyen apenas algunos de los más sonados.
Bogotá, Colombia. "Interbolsa. Empresa criminal de cuello blanco". "El dinero en riesgo se acerca a los 600 mil millones de pesos, perjudicando a unas 812 personas, quienes presentaron unas 1. 112 reclamaciones ante el agente liquidador de la comisionista" (El Universal, 2013).

En todos estos casos la contabilidad estuvo en el ojo del huracán, ya porque hubo falseamiento de la información, o porque hubo ocultamiento, destrucción de documentos y otras acciones fraudulentas. Así, pudieran citarse más casos de escándalos financieros en el mundo (E् Economista.com, 2015), casos en que incluso las víctimas de los fraudes se vieron involucradas en investigaciones fiscales (El Espectador, 2016). Sin embargo, para los propósitos de este trabajo se considera que los citados casos son suficiente evidencia para demostrar que existió un problema común: la debilidad o la ausencia de fidelidad en la información.

La fidelidad de la información, o representación fiel, como la denomina el IFRSen, el Marco Conceptual para la información financiera, consiste en "Representar fielmente los fenómenos que pretende representar" IIFRS-IASB, 2015, Sección 2.14). Esa valiosa característica cualitativa fundamental generadora de confianza pública, a raíz de los escándalos financieros recibió un golpe de tal gravedad que para recuperarse demanda de la disciplina contable y de las organizaciones mucho tiempo y esfuerzos. Pero tales escándalos, en muchos casos, no hubieran ocurrido o se hubiera minimizado su impacto si se hubiera conocido 
un sistema objetivo de evaluación que midiera el grado de fidelidad de la información.

Durante el cuarto lustro del siglo XXI la debilidad en los sistemas de control y aseguramiento de la información no ha sido superada, no obstante que algunos países como Estados Unidos han tomado medidas para la previsión del riesgo, por ejemplo la promulgación de la Ley Sarbanex-Oxley (Díaz Morales, 2005), y en Colombia, la adopción de las Normas Internacionales de Auditoría y las Normas Internacionales de Aseguramiento Ministerio de, Comercio, Industria y Turismo, 2015). Sin embargo, la legislación por sí sola, - las normas emergidas de las sanas prácticas, no han sido suficientemente eficaces para la prevención de los riesgos en la seguridad de la información.

Medir el grado de fidelidad de la información contable fortalecería el trabajo de aseguramiento de la información y permitiría contar con parámetros de confianza entre los usuarios, lo cual sería útil también a las autoridades de fiscalización y en los procesos jurídicos tanto civiles como penales.

En el presente trabajo se propone un modelo para evaluar la fidelidad de la información contable y financiera, con la intención de aportar una respuesta a la necesidad de asegurar la información que difunden las organizaciones y que alimenta el sistema general de información disponible para que los agentes sociales tomen sus decisiones. La propuesta se basa en el principio de autonomía de la información contable. Este principio permite dejar una huella objetiva en el punto mismo de la decisión y constituye pista de auditoría para efectuar evaluaciones más seguras.

Teniendo en cuenta el Marco Conceptual de IFRS, "Los informes financieros representan fenómenos económicos en palabras y números. Para ser útil, la información financiera debe no sólo representar los fenómenos relevantes, sino que también debe representar fielmente los fenómenos que pretende representar" (IFRS Foundation, 2010, p 13).

El problema es entonces, ¿Cómo sería un sistema de medición que permita a los agentes sociales saber si una información contable representa fielmente los fenómenos que pretende representar? Para orientar la solución al problema de investigación se formula la siguiente hipótesis:

\section{HIPÓTESIS}

H1. Un sistema de indicadores de eficacia desarrollado a partir del principio de autonomía de la información permite fortalecer la confianza de los agentes sociales en un conjunto informacional, respecto de su punto de fidelidad.

Se tienen como variables las siguientes:

\begin{tabular}{|l|l|c|}
\multicolumn{1}{|c|}{ Variable } & \multicolumn{1}{c|}{ Clase } & Símbolo \\
\hline Eficacia & Variable dependiente. & E \\
\hline Fidelidad & $\begin{array}{l}\text { Constante, representa } \\
\text { el 100\% de pureza } \\
\text { de la información } \\
\text { representada. }\end{array}$ & 1 \\
\hline $\begin{array}{l}\text { Confianza de los } \\
\text { usuarios de la } \\
\text { información }\end{array}$ & Independiente. \\
\hline $\begin{array}{l}\text { Conjunto o masa } \\
\text { información contable } \\
\text { y financiera }\end{array}$ & $\begin{array}{l}\text { Relativa. Constituye el } \\
\text { objeto de evaluación. }\end{array}$ & icf \\
\hline $\begin{array}{l}\text { Autonomía de la } \\
\text { información }\end{array}$ & $\begin{array}{l}\text { Principio natural con- } \\
\text { tenido en la decisión } \\
\text { delos agentes sociales. }\end{array}$ & Ai \\
\hline
\end{tabular}

3. MARCO CONCEPTUAL

En este trabajo se define la contabilidad como el campo del conocimiento que estudia y da cuenta de los fenómenos patrimoniales (Avellaneda Bautista C. A., Contametría, Ciclo Tecnológico de la Contabilidad, 2016). El presente estudio corresponde al campo de conocimiento contable, enfatizando el área del control y el aseguramiento de la información que la contabilidad genera. 
La fidelidad, que es el núcleo problémico de esta investigación, está definida por el Marco Conceptual IASB-IFRS así:

"La representación fiel supone presentar información sobre la base de la sustancia de los fenómenos económicos en lugar de sobre su forma legal. Esta se define por tres características en la información: completa, neutral, libre de error" (IFRS Foundation, $2015 a$, p 38). De manera que toda relación sobre fidelidad en este trabajo hace referencia al concepto dado por IFRS.

Igualmente, aquí se reconoce al patrimonio como el objeto de estudio de la contabilidad, por ser el conjunto de recursos bióticos y abióticos, materiales e inmateriales que los agentes sociales y los pueblos crean, modifican o construyen a través de su historia, para asegurar la supervivencia y procurarse la identidad, el arraigo, la prosperidad, la sustentabilidad y la heredad.

Para los efectos de esta investigación se entiende por agentes sociales las personas naturales 0 morales (jurídicas) ya sean estos individuales o colectivos; y sean de nivel micro (organizaciones), de nivel macro (entidades estatales), o nivel supra macro lorganizaciones de nivel globalizado, que se relacionan entre sí e interactúan en las comunidades dinamizando con sus decisiones los modos de producción.

Por fenómenos patrimoniales se entiende aquí todos los hechos que resultan de las decisiones de los agentes sociales, como la creación, modificación, extinción, circulación, acumulación, construcción, destrucción, consumo y transformación de la riqueza, los que, por ser fuentes de información, son objeto de estudio de la contabilidad.

\section{CLASIFICACIÓN DEL PATRIMONIOY SUS RELACIONES INTERDISCIPLINARIAS}

En su categoría de fenómeno, el patrimonio puede ser estático o dinámico, conceptos ampliamente conocidos en el lenguaje contable. Por ejemplo, los balances se conocen como estados estáticos y estos representan el patrimonio de las organizaciones o de las personas naturales en un momento determinado. Los estados de resultados muestran la dinámica del patrimonio durante un período de tiempo.

Desde su naturaleza, el patrimonio puede ser biótico o abiótico, teniendo en cuenta los bienes que conforman la riqueza de las personas lagentes sociales).

Son bienes bióticos los ganados, plantíos frutales o de otra índole, y bienes abióticos los minerales. Sin embargo, los bienes también pueden ser conceptuales o intangibles, con valor de riqueza o valores financieros y también son del ámbito de la contabilidad.

Por su relación de propiedad el patrimonio puede ser individual, comunitario, colectivo, social. Por su relación antropológica, puede ser cultural, debido a su estrecha relación con los modos de producción o dinámicas patrimoniales, por las manifestaciones heredadas de las comunidades y por constituir un lenguaje con el que expresa la historia de las organizaciones y de los pueblos (patrimonio económico y patrimonio histórico). Como fenómeno sociológico, los patrimonios expresan las relaciones del individuo en sociedad y promueven el progreso técnico y el progreso social (Bajoit, 2014, pp. 9-42).

Mediante funciones como la medición, valoración, representación y evaluación del patrimonio en función del desarrollo socioeconómico de las organizaciones, de las comunidades y de los pueblos, el individuo se informa y toma decisiones, especialmente para la planeación y el control; igualmente lo hace el Estado para efectos de la redistribución y para aplicar justicia en materia económica, entre otras funciones. Tales funciones son naturales de la contabilidad.

Con la psicología, pudiera decirse que el patrimonio tiene la más vinculante de las relaciones, ya que toda dinámica patrimonial parte de las decisiones de las personas reales y genera información para 
un nuevo proceso de decisión. No en vano se han desarrollado la psicología económica y los estudios sobre la decisión del consumidor (Quintanilla, 2002, p. 97). Esta relación es tan significativa que más adelante se amplía el tema en la sección teoría de la decisión.

Entre la contabilidad y el Derecho existe relación de interdisciplinariedad por medio del patrimonio, pues la contabilidad registra las dinámicas patrimoniales en magnitud y valor, las representa e informa para todos los efectos; evalúa la información para asegurar a la sociedad y a las partes interesadas, sobre las cualidades de un paquete de información (estados financieros u otros informes contables), correspondientes a los hechos del agente social emisor de la misma. Para el Derecho, la contabilidad aporta las pruebas para dirimir los conflictos, ya sea en materia mercantil, fiscal, penal, otras, mediante la representación de patrimonios o de sus dinámicas. Por tanto, se considera apropiado que el concepto de aseguramiento que se emite desde el Derecho tenga relación normativa para la contabilidad, en cuanto sea pertinente.

\section{ASEGURAMIENTO}

En el campo contable, el sistema de medición de la fidelidad de la información que aquí se presenta está enfocado al aseguramiento de la misma; los conceptos que se presentan a continuación contienen también elementos significativos en el aseguramiento desde lo contable y por esta razón se incluyen en la fundamentación de este estudio.

Desde los diccionarios

Diccionario Hispanoamericano de Derecho. "Aseguramiento. Tutela jurídica que adopta algunas medidas que la ley permite, para prevenir futuros daños o periuicios sobre el bien jurídico asegurado". "Asegurar. Proteger de daño a las personas y cosas". (Grupo Latino Editores Ltda., 2008, p. 1631. Como se dijo antes, existe estrecha relación conceptual de aseguramiento, desde el Derecho y desde la contabilidad.
- Diccionario Enciclopédico Lexus. Asegurar. Tranquilizar, infundir confianza; preservar de daño a personas o cosas. (Lexus Editores, 2011 . p. 89). De hecho, la fe pública que otorga el contador público es generadora de confianza pública.

- El Pequeño Larousse. "Aseguramiento. Acción y efecto de asegurar / Asegurar. Garantizar, dejar seguro de la certeza de una cosa; afirmar la certeza de lo que se dice" (Ediciones Larousse, S.A., 2000. p. 11 1). El efecto de la firma de un cuerpo de información contable y financiera es asegurar el grado de fidelidad que el mismo contiene.

Desde lo contable, todo proceso de evaluación lleva implícito el aseguramiento de la información con miras a proteger a los agentes sociales ante futuros daños o periuicios patrimoniales, infundir confianza, afirmar la certeza del conjunto informacional evaluado, y disminuir el riesgo en la toma de decisiones.

\section{EL MARCO LEGAL}

El aseguramiento en Colombia está regulado principalmente por la Ley 1314 de 2009, que en su Artículo $5^{\circ}$ expresa el siguiente concepto:

Para los propósitos de esta Ley, se entiende por normas de aseguramiento de información el sistema compuesto por principios, conceptos, técnicas, interpretaciones y guías, que regulan las calidades personales, el comportamiento, la ejecución del trabajo y los informes de un trabajo de aseguramiento de información. Tales normas se componen de normas éticas, normas de control de calidad de los trabajos, normas de auditoría de información financiera histórica, normas de revisión de información financiera histórica y normas de aseguramiento de información distinta de la anterior". (Congreso de Colombia, 2009, Artículo $5^{\circ} \%$

Con fundamento en el concepto legal, se concluye que todo trabajo de auditoría, y especialmente de 
revisoría fiscal, son trabajos que llevan inherente el aseguramiento de la información; ahora bien, hay algunos trabajos cuyo único objetivo es el aseguramiento.

Se debe tener en cuenta que lo que la ley define no es dado a los terceros definirlo. En consecuencia, en Colombia prima la definición legal. Sin embargo, el Artículo 29 del Código Civil colombiano dice que "las palabras técnicas de toda ciencia o arte se tomarán en el sentido que les den los que profesan la misma ciencia o arte; a menos que aparezca claramente que se han tomado en sentido diverso" (Legis, 2012, p. 8).

Dado que es imposible que la ley pueda prever todos los casos de la realidad del mundo, quienes profesan la técnica, ciencia o arte, pueden, con base en la facultad legal, la lógica y la razón, elaborar definiciones acordes con la intencionalidad o el sentido requerido en determinados casos, teniendo en cuenta la pertinencia, las sanas prácticas y las normas de ética en favor de la sociedad.

\section{EMISORES DE NORMATIVA}

En Colombia son emisores válidos en materia contable $y$, por tanto, también para efectos del aseguramiento de la información, el Estado, los organismos facultados para emitir normativas de obligatorio cumplimiento, y los organismos disciplinarios. Igualmente, en el mismo ámbito se reconocen como normas de recomendable cumplimiento las emitidas por organizaciones profesionales, normas estas que surgen de las sanas prácticas, o que, emergiendo de investigaciones científicas, usualmente son adoptadas como normas de sanas prácticas.

El IAASB (International Auditing and Assurance Standards Board), citado por Sarmiento (2012), define el aseguramiento como

un contrato en el cual un profesional expresa una conclusión con el ánimo de incrementar el grado de confianza de los usuarios potenciales distintos de la parte responsable acerca de los resultados de la evaluación o medición de una materia sujeto contra un criterio.

Comunidad Contable publica en su página (muw.comunidadcontable.com, 2018), en forma de gráfico, una agrupación de las Normas de Aseguramiento de la Información, NAl, para demostrar el predominio normativo en la contabilidad, así:

- Código de Ética para profesionales de la contaduría;

- Normas Internacionales de Auditoría, NIA;

- Normas Internacionales de Control de Calidad, NICC;

- Normas Internacionales de Trabajos de Revisión, NITR;

- Normas Internacionales de trabajos para Atestiguar, ISAE;

- Normas Internacionales de Servicios Relacionados (Comunidad contable.com, 2018).

Como se aprecia en el listado, no están incluidas las normas legales nacionales, como la antes indicada Ley 1314 de 2009, las cuales también cuentan porque son cuerpos regulatorios del aseguramiento de la información.

En consecuencias, el experto evaluador deberá aplicar los métodos ya reconocidos y aceptados como sanas prácticas, obedeciendo las normas legales, o podrá también desarrollar métodos adecuados para llevar a cabo su trabajo mejorando los métodos y sistemas, o bien aportar unos nuevos, siempre y cuando esté en el marco general de un ético ejercicio disciplinar.

\section{DEBATE}

Con base en las facultades legales del contador público, pero más sustentado en los principios y métodos de investigación científica, se propone el siguiente debate. 


\section{SOBRE EL ASEGURAMIENTO}

El experto evaluador deberá aplicar los métodos ya reconocidos y aceptados como sanas prácticas, obedeciendo las normas legales, o podrá también desarrollar métodos adecuados para llevar a cabo su trabajo mejorando los métodos y sistemas, o bien aportar unos nuevos, siempre y cuando esté en el marco general de un ético ejercicio disciplinar.

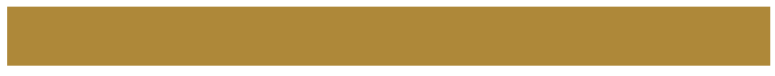

En relación con la línea de tiempo, Mantilla afirma que "el aseguramiento no es predictivo" (Mantilla, 2011 , p. 4). Esta afirmación amerita reflexión pues sabiendo que los hechos ya ocurrieron y conocidos los resultados, el aseguramiento se justifica solamente en virtud de que no todo público tiene acceso a la organización emisora de la información, y por tanto, no es posible evidenciar de manera directa el grado de fidelidad contenido en la misma; ya que el aseguramiento es para "prevenir futuros daños o perjuicios sobre el bien jurídico asegurado", como lo define el Diccionario Hispanoamericano de Derecho (Op. clt.) Sin embargo, el aseguramiento también puede darse de manera concomitante, por la misma razón ya dicha, de manera predictiva como en el caso de información presupuestal, o como el caso de prevención de riesgos, o bien, para el fin teleológico de toda información contable cual es la toma de decisiones: es necesario primero conocer su contenido y después tomar las decisiones.

\section{SOBRE EL PRINCIPIO DE AUTONOMÍA}

Para los efectos de este trabajo, se entiende por autonomía la condición de independencia contenida en los componentes de una decisión, la que, una vez emergida, en su punto de origen no puede ser modificada o eliminada, ni siquiera por el propio decisor. Tal condición constituye entonces un principio natural. Por la definición que antecede, se entiende por autonomía de la información el principio natural inherente a toda decisión relacionada con fenómenos patrimoniales (Avellaneda Bautista C. A., Autonomía de la Información como Principio Contable, 2016$).$

El principio consiste en que: "cuando un agente social toma una decisión relacionada con fenómenos patrimoniales, inherente a ella y aunque el decisor no lo quisiere, existe un conjunto de datos que sirven de insumos a la Contabilidad" (Op. cit., p. 12), datos que son inseparables e 
inmodificables en su punto de origen, ya que, si alguien intentara suprimirlos, desaparecería la decisión.

Debido a su cualidad natural de ser inseparable de la decisión, la autonomía de la información permite un punto de referencia en el cual la fidelidad de la información contable se encuentra con un grado de 100\% de pureza. Luego es a partir de ese punto que se debe indagar en qué parte de la circulación de los datos puede existir contaminación (manipulación) y, si la hubo, entonces se debe determinar en qué grado la fidelidad de la información resulta contaminada. Igualmente se concluye que con metodologías contamétricas como las desarrolladas a partir del principio de autonomía de la información el aseguramiento se puede dar en las tres dimensiones de tiempo: predictivo, concomitante e histórico.

A partir de la autonomía de la información como principio contable (Avellaneda, 2016) se puede diseñar un sistema de evaluación que permita identificar pistas de auditoría de carácter objetivo. Desde allí se puede evaluar la fidelidad de la información contable en cualesquiera de los puntos de circulación de la información, o elaborar pronóstico para anticiparse a los hechos.

El núcleo del sistema aquí propuesto es la decisión como facultad natural del ser humano lagente social), pues en ella se encuentran los elementos fundamentales con que se estructura el objeto de medición; por ello, a continuación se presenta la que para efectos de este trabajo se considera la teoría de la toma de decisión.

\section{TEORÍA DE LA TOMA DE DECISIÓN}

La toma de decisión es el proceso mediante el cual se realiza una elección entre varias opciones o formas para resolver diferentes situaciones de la vida en diferentes contextos. Para dicho proceso se siguen estos pasos: estar conscientes de la situación o acción, analizar posibles alternativas y sus consecuencias, seleccionar la opción pertinente, implementar la decisión y proporcionar retroalimentación (Solano, 2003).

El citado autor resalta cinco factores que caracterizan una decisión: a) Efectos a futuro. b) Reversibilidad, que es la velocidad con que una decisión puede revertirse y la dificultad que implicará ese cambio. c) Impacto. d) Calidad, que se refiere a las relaciones laborales, valores éticos, consideraciones legales, principios básicos de conducta, imagen de la compañía, etc. el Periodicidad, que tiene que ver con la frecuencia con que se toma ese tipo de decisión. Además se resaltan otras características como la información oportuna y suficiente, los conocimientos y la experiencia necesaria, además de la capacidad de análisis y juicio que garantizan un adecuado proceso en la toma de decisiones.

Por tanto, la toma de decisiones se considera una actividad psicológica general y esencial, es una función de la cual puede entenderse toda actividad psicológica y que, a su vez, no puede descomponerse en actividades psicológicas significativas más simples (Madera, Sierra, Andreu, Solar, Álvarez y Morís, 2009). Este proceso es complejo, dada la participación de lo cognitivo (racional) y lo emocional (irracional). La complejidad se deriva también frente a que anatómicamente, involucra diferentes regiones cerebrales y requiere la participación de las funciones ejecutivas. En la teoría de los marcadores somáticos, la emoción guía la conducta de elección, permitiendo que nuestras acciones tengan una previsión de las consecuencias futuras y evitando posibles peligros (Gordillo, Arana, Salvador y Mestas, 20111.

Debido a que, además de la emoción (Martínez, Sánchez, Bechara y Román, 2006), también la toma de decisiones se encuentra asociada a procesos psicológicos complejos como la motivación, la atención, la memoria y procesos cognitivos; se requieren propuestas metodológicas que permitan una medida fiable de la toma de decisiones que delimiten claramente su funcionalidad respecto a otros procesos cognitivos y variables que pudieran estar explicando el proceso de toma de decisiones financieras. 
Los economistas, por ejemplo, están muy condicionados por la noción de elección racional y, como afirma Sen (1993) citado por Quintanilla (2002), la racionalidad es un concepto no aprehensible si no tiene un motivo afín. La racionalidad es, por su naturaleza, una interpretación psicológica que se coloca en la conducta que se observa. Desde la perspectiva de la elección racional, cuando se observa una acción se interpreta que esta acción es el resultado de algunos motivos. El origen de esta interpretación es externo respecto de la conducta que se observa. En realidad, en muchas circunstancias las observaciones de la conducta son bastante pobres (o incluso erróneas) para determinar las elecciones individuales. La información no conductual, tal como la comunicación no verbal, puede ser mucho más reveladora para explicar la motivación individual, especialmente cuando las consideraciones morales dominan la elección (Quintanilla, 2002).

En este sentido, tomar decisiones requiere la participación de las funciones ejecutivas, por ser estas las encargadas de iniciar, supervisar, controlar y evaluar la conducta, el recuerdo de las experiencias pasadas y la valoración de las consecuencias futuras que se integran y procesan a nivel prefrontal con claras implicaciones sociales (Salvador, Mestas, Gordillo, Arana, Meilán, Pérez y Carro, 20101.

La información generada durante el proceso de toma de decisiones también puede estar asociada a relaciones aprendidas y situaciones de experiencias anteriores (Martínez, Sánchez, Bechara y Román, 2006), además de factores asociados al momento en que se produce (Gigerenzer y Murray 1987); en este caso no solo se tiene en cuenta el aprendizaje del individuo sino también del equipo y la organización que toma decisiones (Garzón y Fisher, 2008; Cummings y Worley, 2007, Borda, 2017).

En este contexto, para todas las organizaciones es de vital importancia la toma de decisiones, ya que estas rigen su curso; por eso se debe analizar permanentemente los mecanismos para estudiar y tratar de mejorar las condiciones en que se toman dichas decisiones.
Por otra parte, el conocimiento se materializa y se hace útil en la aplicación de decisiones en donde intervienen elementos como la información, el conocimiento y el modo en que se desarrolla dicho proceso; por tanto, para mejorar el proceso de toma de decisiones se debe asegurar la disposición de estos tres elementos (González, Castro y Roncayo, 2004); una forma de medir la calidad de las decisiones es comparar los resultados de la decisión con las expectativas previas a la toma de esta (Paul Moody, 1983). De igual forma, la toma de decisiones deberá ser distribuida entre las personas y unidades organizativas con un determinado nivel de responsabilidad, otorgándoles autoridad y razones para aceptar sus aciertos y errores (Bueno, 2007).

Como se puede ver, la toma de decisiones involucra muchos intangibles que deben ser intercambiados. Para hacer eso, deben medirse al lado de los elementos tangibles cuyas medidas también deben evaluarse para determinar qué tan bien sirven a los objetivos de quien toma las decisiones.

La medición de los factores intangibles en las decisiones ha desafiado durante mucho tiempo la comprensión humana. El número y la medición son el núcleo de las matemáticas, y las matemáticas son esenciales para la ciencia. La medición numérica debe interpretarse en cuanto a significado y utilidad de acuerdo con su prioridad para servir a los valores en una decisión particular. No tiene la misma prioridad para todos los problemas. Su importancia es relativa, y por tanto, se debe aprender cómo derivar prioridades relativas en la toma de decisiones (Saaty, 2008).

En cuanto a los valores, tanto la comprensión como el juicio y las diferentes formas de pensar permiten tomar decisiones transparentes. En cuanto al juicio, el psicólogo cognitivo Blumenthal (1977), citado por Figuera, Greco y Ehrgott (2005), afirma que para hacer el juicio, una persona debe comparar una impresión inmediata con la impresión en su memoria de estímulos similares; por tanto, usar juicios se ha considerado una práctica cuestionable cuando la objetividad es la norma, y en cuanto a los números el autor muestra que incluso cuando los números se obtienen a 
partir de una escala estándar y se consideran objetivos, su interpretación es siempre subjetiva; entonces se necesita validar la idea de que se puede usar juicios para derivar valores tangibles y así proporcionar mayor credibilidad para usar juicios cuando se trata de intangibles.

Con lo dicho hasta el momento se concluye que una decisión se puede tomar desde cuatro puntos de vista diferentes: los beneficios, la decisión que trae, las oportunidades, los costos que crea o en los que incurre y, finalmente, implica riesgos que podría tener que enfrentar; en este sentido, se puede analizar desde el costo-beneficio y el beneficio-riesgo (Figuera, Greco y Ehrgott, 2005).

\section{POSTULADOS}

Las siguientes proposiciones complementan la base para el modelo contamétrico ${ }^{1}$ evaluativo de la fidelidad de la información contable.

1. Al ser la toma de decisiones un proceso de elección consciente, no cabe duda de que la responsabilidad de los resultados corresponde al tomador de la decisión. En consecuencia, cualquier contaminación que sufra la información contable y financiera en su recorrido por el ciclo de la información, será responsabilidad del decisor, a menos que se demuestre lo contrario.

2. Debido al principio de que una decisión no puede descomponerse en actividades psicológicas más simples, la reversibilidad de una decisión genera un nuevo proceso contamétrico contenido en la decisión reversada.

3. Las consecuencias futuras de una decisión tomada bajo la guía de las emociones y motivaciones es responsabilidad del decisor. Consecuentemente, la contaminación de la información contable y financiera

Contamétrico, en este escrito, hace referencia a las mediciones contables o mediciones realizadas mediante métodos contables. y sus consecuencias, también serán responsabilidad del decisor (Congreso de la República de Colombia, 1995).

4. Dado que la interpretación de los hechos siempre es subjetiva, es necesario que una evaluación al principio de fidelidad enfocada al aseguramiento de la información contable y financiera sea parte de los procedimientos tendientes a verificar lo que debe representar la información (aspecto normativo), para dar paso a la objetividad científica, que contrastará la información evaluada contra la realidad que la misma información pretende representar. Esta condición es necesaria teniendo en cuenta que actualmente la contabilidad está regida por normas emergidas de las sanas prácticas y por normas legales que buscan lo que debe ser, en lugar de enfocarse en los hechos, a lo cual hace referencia el IFRS, en el Marco Conceptual para la Información Financiera, Capitulo 2, Características cualitativas de la información financiera útil, nomenclador 2. 14, "Representación Fiel". (IFRS, Op. cit).

Por las expuestas razones, se propone el sistema de medición de la fidelidad de la información contable a partir del principio de la Autonomía de la Información por ser un principio natural capaz de aportar un mecanismo objetivo para disminuir el riesgo en el aseguramiento de la información contable y financiera.

\section{PROPUESTA DEL SISTEMA PARA MEDIR EL GRADO DE FIDELIDAD CONTENIDO EN UNCUERPOINFORMACIONAL}

En esta sección se sustenta que se puede medir objetivamente el grado de acercamiento que pudiera tener un cuerpo informacional, en relación con el principio de fidelidad. Para el efecto se propone un sistema de medición basado en el principio de autonomía de la información, validado mediante un modelo contamétrico. 


\section{CICLO DE LA INFORMACIÓN}

La entrada al sistema es la decisión de los agentes sociales. El proceso se realiza mediante métodos contamétricos, y la salida es la información contable y financiera que ha de circular hasta llegar a los decisores para la toma de nuevas decisiones, como se explicó en el libro Ciclos de Operaciones Financieras, Concepción contable, del cual se toma el siguiente texto y la figura $\mathrm{N}^{\circ}$ 1, a continuación.

Sea $A(A=$ las decisiones de los agentes sociales) que causa $B$ ( $B=$ los datos para los procesos contables), B causa $C(C=l a$ información contable y financiera resultante de los procesos contables), pero C causa $\mathrm{A}, \mathrm{O}$ mejor, C causa A' (nuevamente, las decisiones de los agentes sociales, pero diferentes a las decisiones que dieron origen al sistema), como se ilustra en la Figura $N^{0} 1$, en la cual, $A$ no es $C$, ni $C$ es $B$, porque $B$ no es $A$, como tampoco $A$ es $A^{\prime}$, pero ilustra claramente la formación del ciclo operacional contable. (Avellaneda B., C.A., Op. cit. p. 77).

\section{Gráfico $\mathbf{N}^{\circ}$ 1. Circularidad del proceso contable.}

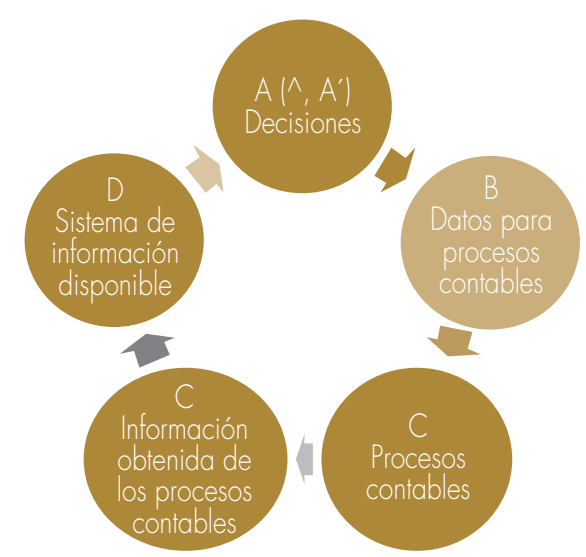

Fuente: Elaboración con base en Avellaneda Bautista, C.A. (2017), Ciclos de Operaciones Financieras, Concepción Contable, p. 78, Bogotá, Universidad Libre.

Con base en la teoría de la decisión antes expuesta, se puede afirmar que solamente las personas como individuos o inmersas en equipos de trabajo y organizaciones reales tienen la facultad natural de tomar decisiones. Se resalta que la toma de decisiones es una actividad psicológica que corresponde a personas en su integralidad y que en ocasiones las decisiones se toman en representación de las organizaciones (personas morales -jurídicas-). Esta conclusión sirve de premisa esencial para la tesis que se describe a continuación.

\section{TESIS}

"Toda decisión relacionada con dinámicas patrimoniales lleva inherente los datos contables que sirven de insumo para los procesos contamétricos" (Avellaneda Bautista, C. A., Ciclos de Operaciones Financieras, concepción contable, 2017, p. 65). Estos procesos convierten los datos en información contable y financiera, la cual, una vez sumada al sistema general de información, queda a disposición de los agentes sociales para toma de nuevas decisiones, cumpliéndose así el ciclo de la información.

\section{EL PRINCIPIO DE AUTONOMÍA DE LA INFORMACIÓN}

La autonomía de la información como principio contable fue presentada en el marco del XXI Congreso Internacional de Contaduría, Administración e Informática, en la Universidad Autónoma de México, UNAM, en octubre de 2016.

\section{Axioma}

La autonomía de la información descansa sobre el siguiente axioma:

"Toda decisión relacionada con dinámicas patrimoniales lleva inherente el dato contable. Emerge con tal autonomía en su punto de origen, que es imposible cualquier acción tendiente a rechazarlo, separarlo, o modificarlo". (Avellaneda Bautista, C. A., Autonomía de la Información como Principio Contable, 2016). 
El principio de autonomía de la información, en su punto de origen, da al dato contable el máximo grado de pureza, razón por la cual esta cualidad permite evaluar y controlar la información contable y financiera con el máximo grado de objetividad. Sin embargo, se debe tener en cuenta que en la medida en que el dato se aleje de su punto de origen la fidelidad, este queda expuesto a riesgos de manipulación contaminante, ya sea por parte del mismo emisor o por parte de agentes diferentes.

Se requiere entonces asegurar la información contra tales riesgos mediante eficaces métodos, procesos y herramientas que, si existen, no son visibles, ni de general conocimiento.

\section{Teorema}

Se propone aquí el siguiente teorema como sustento del modelo para medir fidelidad en un conjunto informacional (o masa de información).

Un sistema que permita evaluar la eficacia (E) en el aseguramiento de la información, puede evidenciar en qué grado tal información se acerca a su punto de fidelidad.

Tal sistema pudiera aportar al menos un indicador de evaluación para relacionar la eficacia con el nivel de confianza del usuario (Cu), y esta confianza en razón de tres factores: la autonomía de la información (Ai), la masa de información contable y financiera representada (icf), y un factor de error permitido (ep).

La razón resultante restada de la unidad evaluable ( $u=1$ ) permite obtener el indicador de fidelidad en la pureza de la información, la cual constituye el grado de aseguramiento; así:

$$
E=1-\frac{C u}{[\text { Ailicf)(ep)] }}
$$

En donde:

Eficacia $=\mathrm{E}$

Fidelidad $=1$ (Por representar el 100\% de pureza en la representación)

Confianza del usuario de la información = Cu

\section{Un sistema que permita \\ evaluar la eficacia (E) en el aseguramiento de la información, puede evidenciar en qué grado tal información se acerca a su punto de fidelidad.}

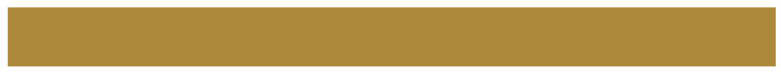


Masa informacional contable $y$ financiera $=$ icf Error permitido $=\mathrm{ep}$

En la siguiente sección se desarrolla el modelo enunciado.

\section{MODELO PARA MEDIR \\ 6. LA EFICACIA EN EL \\ 6. ASEGURAMIENTO DE LA INFORMACIÓN CONTABLE Y FINANCIERA}

Se propone aquí el modelo para evaluar la eficacia en el aseguramiento de la información contable y financiera.

\subsection{CONCEPTO}

Eficacia es la capacidad para lograr el resultado que se espera (Grupo Latino Editores, 2008, p. 7111 . Esta definición satisface la intención pretendida en esta sección.

El aseguramiento. Para los contadores públicos mexicanos son tres los trabajos de aseguramiento: auditorías, revisiones y atestiguamiento.

El concepto es el siguiente:

Los trabajos de aseguramiento son aquellos en los cuales un Contador Público expresa una conclusión diseñada para incrementar el grado de confianza de los usuarios sobre el resultado de la evaluación o medición del asunto sobre el que se realizará el trabajo, con los criterios aplicados. Estos trabajos pueden tener dos enfoques: a) trabajos que proporcionan una seguridad razonable y b) trabajos que proporcionan una seguridad moderada. (Ramírez Torres, 2013 , pp. 4, 5).

También afirma el citado autor que:

el objetivo de un trabajo de seguridad razonable es la reducción del riesgo a un nivel bajo que sea aceptable en las circunstancias del trabajo base para expresar en forma positiva la conclusión del Contador Público, es decir, se emite una opinión. (p. 5).

Tanto el concepto de seguridad razonable como el de aseguramiento se aceptan aquí como ámbito para el modelo que se propone.

De otra parte, de acuerdo con las características contenidas en el marco conceptual de las Normas Internacionales de Información Financiera (NIIIF), la información debe ser fiable, es decir, "libre de error material y de sesgo o prejuicio, y los usuarios pueden confiar en que es la imagen fiel de lo que pretende representar, o de lo que puede esperarse razonablemente que represente" (IASB, 2004, p. 63). Este marco conceptual fue revisado en el año 2010 y a partir de entonces, para el mismo predicado, se hace referencia a la fidelidad y no a la fiabilidad.

\subsection{EL MODELO, SUS VARIABLES Y RELACIONES}

Las siguientes características, variables y relaciones, observadas en las fuentes citadas, conforman el modelo propuesto. 


\section{Tabla 2. Modelo para evaluar la eficacia en el aseguramiento de la información contable y financiera.}

\begin{tabular}{|c|c|}
\hline $\begin{array}{l}\text { Características } \\
\text { del modelo }\end{array}$ & Variables, relaciones y modelo de evaluación \\
\hline $\begin{array}{l}\text { Objetivo del } \\
\text { aseguramiento }\end{array}$ & La fidelidad de la información (Fi). \\
\hline Factor de fiabilidad & $\begin{array}{l}\text { La autonomía de la información }(A i) \text {, es factor determinante en la fidelidad de la } \\
\text { información, entonces su relación es: Ai (Fi). En general, Fi f Ai. La fidelidad de la } \\
\text { información está en función de la autonomía de la información. }\end{array}$ \\
\hline Finalidad & $\begin{array}{l}\text { Se espera tener el más alto grado de confianza de los usuarios (Cu). Esta confianza } \\
\text { está en relación directa con la fidelidad de la información como imagen fiel de lo } \\
\text { que razonablemente represente, o de lo que pretende representar. Su relación es: } \\
\text { Cu f Fi Confianza del usuario, en función de la fidelidad de la información. } \\
\text { Dicho de otro modo: la confianza del usuario (Cu), está en relación directa con } \\
\text { la fidelidad de la información (Fi), teniendo en cuenta que la fidelidad tiene como } \\
\text { factor Ai (factor de autonomía de la información), es decir, que se forma la ecuación: } \\
\qquad \mathrm{Cu}=(\mathrm{Ai}) \text { (Fi) }\end{array}$ \\
\hline $\begin{array}{l}\text { Relación confianza } \\
\text { del usuario/grado de } \\
\text { representación del objeto } \\
\text { representado }\end{array}$ & $\begin{array}{l}\text { La confianza del usuario (Cu) también está en función directa con el grado de } \\
\text { representación objetiva que tenga el objeto representado (Gro). En este caso, la } \\
\text { relación es: } \\
\qquad \begin{array}{l}\text { Cu }=(G r o)\end{array}\end{array}$ \\
\hline $\begin{array}{l}\text { Confianza del usuario en } \\
\text { función de la fidelidad y } \\
\text { del grado de representación } \\
\text { que alcanza el objeto } \\
\text { representado }\end{array}$ & $\begin{array}{l}\text { Combinando las ecuaciones en c) y d), se tiene que la confianza del usuario (Cu) } \\
\text { depende de la información (Ai) y del grado de representación del objeto (Gro). Es } \\
\text { decir, Cu es una variable dependiente de } \mathrm{Ai}^{*} \mathrm{Fi}^{*} \text { Gro, y por tanto, resulta la ecuación: } \\
\qquad \mathrm{Cu}=\text { (Ai) (Fi) (Gro) }\end{array}$ \\
\hline $\begin{array}{l}\text { Relación de la variable } \\
\text { eficacia, en función de las } \\
\text { variables que determinan la } \\
\text { confianza del usuario }\end{array}$ & $\begin{array}{l}\text { El objetivo propuesto es construir un modelo para evaluar la eficacia (E) del } \\
\text { aseguramiento de la información. Es decir, se trata de representar un procedimiento } \\
\text { general que permita conocer el grado de acercamiento a la fidelidad que tiene un } \\
\text { cuerpo de información contable (icf) con los hechos que pretende representar, para } \\
\text { que se pueda conceptuar como fiel y por tal razón genere confianza. } \\
\text { Para representar el grado de eficacia (E) en el aseguramiento de la información, se } \\
\text { deben conocer las magnitudes de Fi, Ai y de Gro. } \\
\text { Como las magnitudes son medidas, entonces se pueden representar como variables y } \\
\text { con ellas medir la confianza del usuario (Cu) (ver ecuación en la celda e). Por tanto: } \\
\qquad \mathrm{Cu}=\{\mathrm{E}[\mathrm{Ai})(\mathrm{Fi})(\mathrm{Gro})]\}\end{array}$ \\
\hline $\begin{array}{l}\text { Introducción de la variable } \\
\text { Eficacia en las funciones de } \\
\text { confianza del usuario. }\end{array}$ & $\begin{array}{l}\text { Comolo que se quiere es conocer el grado de eficacia, explicitamos esa variable } \\
\text { como dependiente de los factores que forman Cu y le incorporamos el factor de } \\
\text { error permitido (ep): } \\
\qquad E=\frac{C u}{[A i(e p) /(i c f)]} \\
\text { El resultado de este modelo será el grado de confianza que el usuario de la } \\
\text { información deposite en la misma. Dicho de otra manera, es el grado de seguridad } \\
\text { que la información proporciona al usuario de esta. }\end{array}$ \\
\hline
\end{tabular}




\section{Características} del modelo

\section{Variables, relaciones y modelo de evaluación}

Criterios para determinar La medición de eficacia tiene valor máximo el 100 \%; sin embargo, en la práctica el índice de eficacia en es casi imposible alcanzarlo, ya porque los procedimientos de medición no alcanzan el aseguramiento de la el grado absoluto de precisión, porque se utilizan herramientas estadísticas que no información lo grado de lo permiten, o porque, si bien los datos en el origen alcanzan su máxima pureza fidelidad). debido al principio de autonomía de la información, a partir de ese momento están expuestos a diferentes formas de contaminación que pueden restarles pureza. Es necesario, entonces, estimar un grado razonable de impurezas, que permita al usuario tener relativa confianza en la información.

- Cada evaluador determinará su propio índice de impureza aceptable de acuerdo con las circunstancias.

- Para validar este modelo se aceptará un factor de 0,04 de impurezas en el objeto por evaluar (objeto = conjunto de información contable y financiera -icf1, factor que constituye el error permitido (ep). El principio de autonomía de la información (Ai), tiene por valor 100\%, debido a que es un principio natural autónomo, independiente de la voluntad del agente decisor.

- El factor ideal de fidelidad de la información (Fi), es también de 100\%.

- El grado ideal de representación del objeto que se quiere representar (Gro) también es de $100 \%$.

- Debido a la relatividad en la precisión de la información, el grado de confianza del usuario será también relativo, y por tanto, su índice estará en un rango entre 0 y 100. Cuando no se confía en la información = 0; total confianza = 100. Valores intermedios en este rango indicarán el grado de confianza que la información genera en el usuario (o grado de fidelidad de la información).

Determinación de la identidad contra la cual medir la eficacia en el aseguramiento de la información.
Utilizando el artificio algebraico de escribir el factor 1 que está presente como factor de toda variable y despejando en la ecuación de la celda d), tenemos la constante que sirve de identidad o límite de valor máximo que puede tomar el resultado de la medición de eficacia.

$$
\text { d) } 1(\mathrm{Cu})=1(\mathrm{Gro})
$$

Entonces: $1=\frac{\text { gro. }}{C_{u}}$; así como también: $1=\frac{C u}{\text { gro }}$

Determinación del índice de eficacia del aseguramiento de la información, con base en el principio de autonomía de la información (Ai)

Tomando la ecuación de la celda gl e incorporando la magnitud identidad = 1, a cuyo valor se suma algebraicamente el resultado de operar los elementos variables, se tiene:

$$
E=1-\frac{C u}{[\text { Ailep)/(icf)] }}
$$

Prueba del indicador de eficacia en el aseguramiento de la información.
Para validación, se pone a prueba solamente la ecuación de la celda i), con los valores de la celda h) que sean pertinentes:

$$
E=1-\frac{C u}{[\text { Ai(ep)/icf })]}
$$




\begin{tabular}{|c|c|}
\hline $\begin{array}{l}\text { Características } \\
\text { del modelo }\end{array}$ & Variables, relaciones y modelo de evaluación \\
\hline & $\begin{array}{l}\text { Remplazando: } \\
\qquad E=1-\frac{100}{[100(.04)(100)]} \\
\text { Resolviendo aritméticamente: } E=1-\frac{1}{[25]} \\
\qquad E=1-0,04 \text {; entonces } E=0,96 \\
\text { Indicador que razonablemente es aceptable. }\end{array}$ \\
\hline $\begin{array}{l}\text { Si el error permitido fuera } \\
0,07\end{array}$ & $\begin{array}{l}\text { La ecuación sería: } E=1-\frac{1}{[7]} \\
\qquad \text { Resolviendo: } E=1-0,1429 \\
\text { Entonces: } E=1-0,1429 \text {; por tanto, } E=0,86 \\
\text { El evaluador decidirá si acepta o no este grado de eficacia en la seguridad de la } \\
\text { información. } \\
\text { En cuanto al usuario de la información, posiblemente, debido al principio de } \\
\text { incertidumbre que reina en el mundo real, sea aceptado el grado de seguridad de } \\
\text { la información evaluada. }\end{array}$ \\
\hline
\end{tabular}

Fuente: Elaboración propia, basada en Avellaneda, B. (2017), "Autonomía de la Información Contable, Principio Eficaz para Aseguramiento y Control". Ponencia en el marco del $4^{\circ}$. Congreso Transatlántico de Contabilidad, Auditoría, Control de Gestión y Gestión de Costos". Lyon, Francia, Université Jean Moulin, 22 y 23 de junio de 2017, Lyon 3.

7. RESULTADOS

1. Se diseñó el modelo para evaluar la eficacia en el aseguramiento de la información y se puso a prueba la ecuación que genera el indicador, con lo cual queda demostrada la hipótesis.

2. En el modelo para evaluar la eficacia en aseguramiento de la información, cada variable está en función de las demás del sistema y cada una tiene su propia ecuación.

3. El indicador hallado en la prueba es de 0.96 , que equivale a $96 \%$ de eficacia en una evaluación en donde se tienen en cuenta las magnitudes máximas, que corresponden a $100 \%$, teniendo en cuenta un error permitido de 0,04. Se considera un excelente indicador de evaluación, y podrá generar un alto grado de confianza en el usuario de la información contable y financiera. Sin embargo, un evaluador de eficacia de aseguramiento de la información puede encontrar en sus procedimientos que el error aceptable es menor o mayor que el utilizado en el modelo $y$, por tanto, el indicador será diferente lejemplo $=0,86$, en celda kl). Igualmente, si considera que no le pueden dar 100\% del peso a las otras variables, en el mismo sentido el indicador se moverá hacia arriba (mejorando el indicador de eficacia) o hacia abajo (haciéndolo más débill; es decir, que lo representado en el cuerpo informacional se acerca o se aleja del punto máximo de fidelidad; en el mismo sentido será la confianza del usuario. 
4. Vale advertir que la confianza del usuario, en el sentido negativo varía en forma geométrica en tanto la medición de eficacia varía en forma aritmética; y en el sentido positivo la confianza varía en forma aritmética. Esto es debido al principio de prudencia, inherente a los agentes decisores.

\section{CONCLUSIONES}

Por el creciente nivel de corrupción a nivel mundial, especialmente en lo que concierne a fraudes en el patrimonio de los agentes sociales, hechos evidenciados por los grandes escándalos financieros ocurridos durante los recientes cinco lustros en los que se destaca la manipulación de la información contable y financiera y con tal manipulación se falseó la fidelidad, se hace necesario fortalecer los sistemas de aseguramiento.

El principio de autonomía de la información consiste en una dinámica de origen natural, según la cual el dato contable emerge como parte esencial de la decisión de los agentes sociales. En su punto de origen el dato tiene tal autonomía que no puede modificarse, eliminar, o ignorar, pues un intento destruiría la decisión que es punto de origen de los datos contables que emergen de una facultad natural, pero principalmente por su capacidad autónoma de existir en la esencia de la decisión, constituye un punto verdaderamente objetivo por tener en cuenta en la evaluación de la fidelidad, porque en ese punto el grado de fidelidad alcanza su máxima pureza.

La existencia de autonomía de la información contable como principio natural, fue demostrada recientemente (UNAM, octubre de 2016), encontrando entre sus propiedades que es independiente de lo regulado por normas emergidas de las sanas prácticas y de la regulación jurídica, es de origen natural y la autonomía de su existencia puede llegar a ser soporte científico en los procesos de aseguramiento de la información contable y financiera, para fortalecer la confianza pública, como se ha propuesto en el presente trabajo.
Sobre la base del principio de autonomía de la información contable, se construyó el modelo que permite disponer de indicadores de eficacia para medir o evaluar objetivamente el grado de acercamiento o alejamiento que pueda tener un cuerpo informacional hacia el punto de pureza de fidelidad. Es decir, qué tanto esa información representa lo que pretende representar. Del modelo aquí propuesto se pueden derivar herramientas evaluativas en muchos sentidos y nuevas investigaciones, pero lo significativo del mismo es que cualquiera sea la información contable y financiera, ella cuenta con un punto de origen cierto en el que emerge con total pureza. Ese hecho permite disponer del punto de referencia que la sociedad requiere para asegurar la información, prevenir riesgos de adulteración o encontrar los puntos en donde el objeto de evaluación ha sido adulterado. Es decir, permite un aseguramiento objetivo de la información contable y financiera.

Como resultado del sistema se obtuvo un indicador de eficacia para medir el grado de acercamiento que pueda tener un cuerpo de información contable y financiera a la característica de la fidelidad que la misma información debe cumplir. Se convalidó poniéndolo a prueba en dos ejemplos que se incorporaron en este artículo. Se pretende que los resultados de su adecuada aplicación aporten fortalecimiento para la generación de confianza tanto

mercantil como jurídica y fiscal, en la información que publican las organizaciones, a la vez que se recupere la confianza pública en la contabilidad.

\section{REFERENCIAS}

Avellaneda Bautista, C. A. (2016). Autonomía de la Información como Principio Contable. En: U. A. UNAM, Memorias del XXI Congreso Internacional de Contaduría, Administración e Informática (pp. Sección 5.02. Disponible en: http://premio.investiga.fca.unam.mx/ docs/XXI/7. 1.pdf). México, : UNAM.

Avellaneda Bautista, C. A. (2016). Contametría, Ciclo Tecnológico de la Contabilidad. En: Memorias del Tercer Simposio Internacional de Contametría. Bogotá, D.C.: Universidad Libre. 
Avellaneda Bautista, C. A. (2017). Ciclos de Operaciones Financieras, concepción contable. Bogotá, D.C.: Universidad Libre.

Bajoit, G. (2014). Relaciones de clase y Modos de Producción. Teoría y Análisis. Cultura y representciones sociales. Año 9, No. 17.

Bueno, E. (2007). Propuesta de documento marco: El nuevo modelo de empresa y de su gobierno en la economía actual. Comisión de Organización y Sistemas AECA. Recuperado de: http://www.aeca. es/comisiones/organizacionysistemas/ propuestadocumentomarco-feb07.pdf

Borda, I. (2017). Validación de una escala de los factores constitutivos de los tres niveles del conocimiento en las organizaciones. Revista Internacional Administración y Finanzas. Vol. 10, No. 1, 2017, pp. 95-108.

Cummings, T., y Worley, C. (2007). Desarrollo organizacional y cambio ( $8^{a}$ ed.) México: Thomson Editores S.A.

Figuera, J.; Greco, S., and Ehrgott, M. (eds) (2005). Multiple Criteria Decision Analysis, State of the Art Surveys. New York: Springer.

Caracol.com/Radio (25 de febrero de 201 1). hitp:// caracol.com.co/radio/201 1/02/25/ judicial/1298656860 431573.html. Obtenido de: Cronología del 'Carrusel de la contratación'en Bogotá: http:// caracol.com.co/radio/20 1 1/02/25/ judicial/1298656860 431573.html

Comunidad contable.com (s/f). Estas son las normas de Aseguramiento de la Información NAl. Obtenido de: wnw. comunidadcontable. com: http://www. comunidadcontable. comBancoconocimiento/normas-deaseguramiento-de-la-informacion-

Congreso de Colombia 13 de julio de 2009, Artículo 5\%. Ley 1314 de 2009. Por la cual se regulan los principios y normas de contabilidad, información financiera y de aseguramiento de información. Bogotá, D. C., República de Colombia: Gobierno Nacional.
Congreso de la República de Colombia (1995). Ley 222 de 1995. Ley 222 de 1995. República de Colombia: Congreso de la República.

Corrigan, T., \& Lewis, W. (1998). Escándalo del Banco Morgan. Nueva York: El Tiempo.com.

Díaz Morales, J. (2005). La Ley Sarbanex-Oxley y la Auditoría. Revista Partida Doble. No 169 104-109.

Ediciones Larousse, S.A. (2000). El Pequeño Larousse llustrado. Bogotá, D.C.: Ediciones Larousse de Colombia, Ltda.

El Economista .com (11 de febrero de 2015). Los mayores escándalos financieros de los últimos años. El Economista. Disponible en: http://eleconomistacom.mx/fondos/ 2015/02/11/mayores-escándalos-financieros-ultimos-años.

El Espectador (14 de noviembre de 2016). La DIAN persigue a víctima de estafa financiera. El Espectador. Disponible en: http://blogs. elespectador.com/actualidad/detras-de-interbolsa/la-dian-persigue víctima-estafa-financiera.

El País (21 de enero de 2004). Crisis de Confianza. Obtenido de: hitps://cincodias.elpais.com/ cincodias/2004/01/21/empresas/10746 95979850215 html: htpps://cincodias.elpais. com/cincodias/2004/01/21 empresas/107

$4695979850215 . \mathrm{html}$

El País.es (05 de julio de 2006). El caso Enron. Obtenido de: https: / / economia.elpais.com/ economia/2006/07/05/actualidad/1 15

2084782_850215.html: https://economia. elpais.com/economia/2006/07/05/ actualidad/ 1 152084782_850215.html

El Universal, Cartagena (2013). Interbolsa, empresa criminal de cuello blanco. Obtenido de: wnw. eluniversal.com: @eluniversalctg.com.co

Garzón, M., y Fisher, A. 2008. Modelo teórico de aprendizaje organizacional. Pensamiento y gestión, 24, 195-224. 
Gigerenzer, G., y Murray, D. J. (1987). Cognition as intuitive statistics. Hillsdale, NJ: Erlbaum.

González, A.; Castro, J., y Roncayo, M. (2004). Diagnóstico de la gestión del conocimiento en una empresa grande en la ciudad de Barranquilla (Colombia): una actividad de vinculación cooperativa. Revista Ingeniería y Desarrollo, 16, 70- 103.

Gordillo, F.; Arana, J.; Salvador, J., y Mestas, L. (201 1). Emoción y toma de decisiones: teoría y aplicación de la lowa Gambling Task. Revista Electrónica de Psicología Iztacala, $14,(1)$.

Grupo Latino Editores Ltda. (2008). Diccionario Hispanoamericano de Derecho. Bogotá, D.C.: Grupo Latino Editores.

IASB (2004). Normas Inernacionales de Información Financiera-Marco Conceptuat. London: IASB.

IFRS Foundation (2010). Marco Conceptual para la información financiera. IFRS Foundation.

IFRS-IASB (mayo de 2015, Sección 2. 14). http:// www.ifrs.org/-/media/project/conceptualframework/exposure-draft/publisheddocuments/ed-conceptual-framework-es.pdf. Obtenido de hitp://www.ifrs.org/-/media/ project/conceptual-framework/exposuredraft/published-documents/ed-conceptualframework-es.pdf

Legis (2012). Código Civil, 28 ed. Bogotá: Legis Editores S. A.

Lexus Editores (2011). Diccionario Enciclopédico Lexus Color. Barcelona, España: Lexus Editores.

Mantilla, S. A. 11 de junio de 201 1). Aseguramiento: ¿Qué es eso? Obtenido de: https://issuu.com/samantilla/docs/que_ es_aseguramiento: https://issuu.com/ samantilla/docs/que_es_aseguramiento

Madera, I.; Sierra, S.; Andreu, F.; Solar, P.; Álvarez, I., y Morís, J. (2009). La toma de decisiones como proceso psicológico general: la supuesta excepción de la psicología experimental del condicionamiento. Revista de Historia de la Psicología, 30 (2-3), 195 -206.

Martínez, S.; Sánchez, N.; Bechara, A., y Román, F. (2006). Mecanismos cerebrales de la toma de decisiones. Revista de Neurología, 42(7), $411-418$.

Ministerio de Comercio, Industria y Turismo (20 de febrero de 2015). Decreto 302, Reglamentario de la Ley 1314 de 2009. Obtenido de: Ministerio de Comercio, Industria y Turismo: www.mincit.gov.co/loader.php?/ servicio=documentos... Decreto 302-2015

Moody, P. (1983). Decision making: methods for better decisions. New York: McGraw-Hill.

EFE. El Tiempo (9 de julio de 2002). http:// www.eltiempo.com/archivo/documento/ mam-1325075. Obtenido de: Merk: otro escándalo contable: http://www.eltiempo. com/archivo/documento/mam-1325075

Quintanilla, I. (2002). Daniel Kahneman y la Psicología Económica. Revista de la Psicología del Trabajo y de las organizaciones, $N^{0} 18$, 95-108.

Ramírez Torres, O. J. (2013). Normatividad en México. Su armonización y aplicación. Revista Contaduría Pública. Instituto Mexicano de Contadores Públicos, 1-7.

Saaty, T. (2008). Decision making with the analytic hierarchy process. Int. J. Services Sciences, 1, (1).

Salvador, J.; Mestas, L.; Gordillo, F.; Arana, J. Meilán; Pérez, E., y Carro, J. (2010). Toma de decisiones en la anorexia nerviosa. Revista de Neurología, 50, 703-704.

Sarmiento Pavas, D. (2012). Aplicación de las Normas Internacionales de Aseguramiento en Colombia. Obtenido de: http://www. javeriana.edu.co/personales/hbermude/ audire/dsp.pdf: http://www.javeriana.edu. co/personales/hbermude/audire/dsp.pdf

Solano, Ana Isabel (2003). Toma de decisiones gerenciales. Tecnología en Marcha, 16, (3). 